\title{
Wireless Relay Communications using an Unmanned Aerial Vehicle
}

\author{
Pengcheng Zhan, Kai Yu, A. Lee Swindlehurst \\ Department of Electrical and Computer Engineering, \\ Brigham Young University, Provo, UT, USA, 84602 \\ Telephone: (801) 422-3930, Fax: (801) 422-0201 \\ e-mail: $\{$ pz8,kaiyu,swindle $\} @$ ee.byu.edu
}

\begin{abstract}
Herein, we investigate the optimal deployment of an unmanned aerial vehicle (UAV) in a wireless relay communication system. The optimal UAV position is found by maximizing the average data rate, while at the same time keeping the symbol error rate (SER) below a certain threshold. We derive a closedform expression for the average data rate in a fixed wireless link using adaptive modulation. By using the alternate definite integral form for the Gaussian Q-function, the symbol error rate (SER) of the system in the link level is evaluated. An upper bound on the SER is also derived using the improved exponential bounds for the Q-function. It is shown that the derived SER expression matches the simulation results very well and the derived upper bound is tight for a wide range of SNRs. Simulation results also show that the system data rate matches the derived closed-form expression.
\end{abstract}

\section{INTRODUCTION}

Recently, unmanned aerial vehicles (UAVs) have attracted considerable attention in many military as well as civilian applications [1], [2]. Besides other advantages, one major advantage of using UAVs is that they can be quickly deployed into the battlefield or various communication environments as relays [3], and therefore improve the performance of wireless communications systems [4], [5]. For example, in many communication scenarios, there exist obstacles (such as mountains, buildings, etc) that severely deteriorate or even block the signal between the transmitter and the receiver. In such cases, one can deploy a UAV to help setup the communication link and improve the communication performance, i.e. using the UAV as a relay between the transmitter and the receiver.

In this paper, we assume there is no direct communication link between the transmitter and the receiver. A UAV is assumed to be positioned so that it can relay messages from the transmitter to the receiver, as depicted in Fig. 1. Assuming adaptive modulation is employed in the communication system, we analyze the average data rate of the system, and investigate the optimal position of the UAV so that the transmission rate is maximized under the constraint that the symbol error rate (SER) is below a certain threshold.

The paper is organized as follows. Section II presents the system model used in this paper. In Section III, we derive the closed-form expression of the average data rate, and formulate the optimization problem to find the optimal position of the

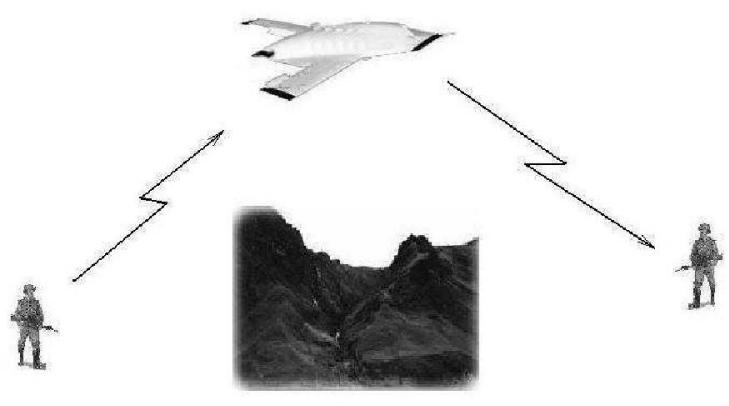

Fig. 1. Battlefield relay communication scenario

UAV. An SER analysis is also given in this section. Numerical simulation results are presented in Section IV, followed by some conclusions in Section V.

\section{System Model}

\section{A. Two-hop half duplex protocal}

In this paper, we assume that a two-hop half-duplex protocol is used in the system. During the first time slot, the transmitter sends the desired message to the UAV. The UAV decodes the message and then sends it to the receiver in the second time slot. Note that we assume the UAV can not transmit and receive simultaneously.

Using the above transmission protocol, the signal model for the first and second time slots can be written as

$$
\mathbf{y}_{1}=\sqrt{\frac{E_{s 1}}{M}} \mathbf{H}_{1} \mathbf{s}_{1}+\mathbf{n}_{1}
$$

and

$$
\mathbf{y}_{2}=\sqrt{\frac{E_{s 2}}{N}} \mathbf{H}_{2} \mathbf{s}_{2}+\mathbf{n}_{2},
$$

where $\mathbf{y}_{1}$ is the received signal at the $\mathrm{UAV}, \mathbf{H}_{1}$ is the channel matrix between the transmitter and the UAV, $s_{1}$ is the transmitted signal, and $\mathbf{n}_{1}$ is additive noise. Similar definitions are used in (2), except that in this time slot the UAV becomes the transmitter. We will let $M$ denote the number of antennas at ground stations, $N$ the number of antennas at UAV, and we 
let $E_{s 1}, E_{s 2}$ represent the transmission power during the two time slots.

\section{B. Channel model}

We assume the channels $\mathbf{H}_{1}$ and $\mathbf{H}_{2}$ are Rayleigh fading channels with large scale path loss, i.e.

$$
\mathbf{H}_{i}=\frac{\mathbf{H}_{n o r m}}{d_{i, u}^{\alpha}},
$$

where $\mathbf{H}_{\text {norm }}$ is a normalized complex Gaussian channel which when stacked in an $N M \times 1$ vector has the distribution $\mathcal{C N}\left(\mathbf{0}, \mathbf{R}_{H}\right)$, and $d_{i, u}$ is the distance between the $i$ th access point (AP) $\{i=1,2\}$ and the UAV. In our application, AP1 is the transmitter and AP2 the receiver. For free space transmission, the path-loss exponent $\alpha$ equals one [6]. Values of $\alpha>1$ occur in obstructed environments, while $\alpha<1$ is common in wave-guided environments. Note that log-normal shadow fading can easily be included in the channel model and the analysis below. Assume the coordinates of the $i$ th AP and UAV are given as $\left[\begin{array}{lll}x_{i} & y_{i} & h_{i}\end{array}\right]^{T}$ and $\left[\begin{array}{lll}x_{u} & y_{u} & h_{u}\end{array}\right]^{T}$ respectively, so that $d_{i, u}$ can be written as

$$
d_{i, u}=\sqrt{\left(x_{u}-x_{i}\right)^{2}+\left(y_{u}-y_{i}\right)^{2}+\left(h_{u}-h_{i}\right)^{2}} .
$$

We use the well known Kronecker approach [7], [8] to model the correlation matrix $\mathbf{R}_{H}$ of the MIMO wireless channels, i.e., $\mathbf{R}_{H}=\mathbf{R}_{T x} \otimes \mathbf{R}_{R x}$, where $\mathbf{R}_{T x}$ are $\mathbf{R}_{R x}$ are respectively the normalized transmit and receive channel correlation matrices. If AP1 and AP2 are located in multipath scattering environments, we would see low spatial correlation at AP1 and AP2. At the UAV side, however, high spatial correlation is expected since there are few if any scatterers near the UAV. The normalized channel matrix is expressed as

$$
\mathbf{H}_{\text {norm }}=\left(\mathbf{R}_{R x}\right)^{1 / 2} \mathbf{G}\left[\left(\mathbf{R}_{T x}\right)^{1 / 2}\right]^{T},
$$

where the stochastic $N$ by $M$ matrix $\mathbf{G}$ contains independent and identically distributed (IID) $\mathcal{C N}(0,1)$ elements, $(\cdot)^{T}$ denotes transpose, $(\cdot)^{1 / 2}$ is defined such that $\mathbf{R}^{1 / 2}\left(\mathbf{R}^{1 / 2}\right)^{H}=$ $\mathbf{R}$, and $(\cdot)^{H}$ is the Hermitian transpose.

\section{Adaptive modulation}

We assume that the system employs adaptive modulation based on the current channel SNR, denoted by $\gamma$. For a given desired SER, the required SNR thresholds are predetermined using the SER expression given in [9], [10]:

$$
P_{e} \approx \bar{N}_{e} Q\left(\sqrt{\frac{\gamma d_{\min }^{2}}{2}}\right),
$$

where $P_{e}$ is the symbol error probability, $\bar{N}_{e}$ is the number of nearest neighbor constellation points, and $d_{\min }$ is the minimum separation distance between points on the underlying constellation.

Assume that $\gamma_{k}$ and $\gamma_{k+1}$ are the predetermined SNR thresholds for the $k$ th and $(k+1)$ th modulation schemes respectively. If $\gamma_{k+1}>\gamma \geq \gamma_{k}$, the $k$ th modulation scheme will be used to transmit the message. If $\gamma<\gamma_{1}$, no transmit scheme will be chosen, which indicates there will be no transmission between the transmitter and the receiver.

\section{Orthogonal space-time block coding}

We assume that in both time slots, only the receiver knows the channel matrix. Hence, orthogonal space-time block codes (OSTBC) [11] are used to transmit the data. For example, in the $2 \times 2$ case, the well-known Alamouti code [12] is employed. Since adaptive modulation is used, the receiver needs to determine/predict a suitable modulation scheme and feed this information back to the transmitter. In this paper, we assume that this feedback is perfect, i.e. the transmitter knows which modulation scheme to use. Note that feeding back the modulation scheme costs much less than feeding back the full channel state information.

\section{System Analysis}

OSTBC exploits the diversity of the MIMO channels, and the instantaneous uplink SNR at the UAV can be expressed as

$$
\begin{aligned}
\gamma & =\left\|\mathbf{H}_{1}\right\|_{F}^{2} \frac{E_{s 1}}{M \sigma_{N}^{2}} \\
& =\left\|\mathbf{H}_{1}\right\|_{F}^{2} \rho,
\end{aligned}
$$

where $\rho$ is defined as $\rho=\frac{E_{s 1}}{M \sigma_{N}^{2}}, \sigma_{N}^{2}$ is the noise power, and $\|\cdot\|_{F}$ denotes the Frobenius norm. Plugging (3) into (7), we obtain

$$
\gamma=\frac{\left\|\mathbf{H}_{\text {norm }}\right\|_{F}^{2}}{d_{1, u}^{2 \alpha}} \rho .
$$

A similar analysis can be used to find the SNR of the downlink channel from the UAV, if we replace $d_{1, u}^{2 \alpha}$ with $d_{2, u}^{2 \alpha}$, and $M$ with $N$

In [13], using the inverse Laplace transform, the probability density function (PDF) of $\left\|\mathbf{H}_{\text {norm }}\right\|_{F}^{2}$ is derived as

$$
f(x)=\sum_{j=1}^{P} \sum_{k=1}^{m_{j}} A_{j k} \frac{x^{k-1}}{(k-1) ! \sigma_{j}^{k}} e^{-\frac{x}{\sigma_{j}}} u(x),
$$

where $\sigma_{j}(j=1,2, \cdots, P)$ are the distinct non-zero eigenvalues of $\mathbf{R}_{H}$, and $m_{j}$ denotes the respective multiplicities of $\sigma_{j}$. By solving a system of linear equations, $A_{j k}$ can be determined [13]. By defining

$$
\begin{aligned}
g(n, \alpha, x) & =\int \frac{x^{n}}{n !} e^{\alpha x} d x \\
& =\frac{1}{n !} \frac{e^{\alpha x}}{\alpha^{n+1}} \sum_{i=0}^{n}(-1)^{n-i} \frac{n !}{i !}(\alpha x)^{i},
\end{aligned}
$$

the cumulative distribution function (CDF) of $\left\|\mathbf{H}_{\text {norm }}\right\|_{F}^{2}$ can be expressed as in (11).

\section{A. Ergodic normalized transmission rate}

Due to the random nature of the channel matrices, the instantaneous transmission rate is different for different channel realizations. Therefore, we define the ergodic normalized transmission rate (ENTR) and use it as the criteria to quantify the performance of the link. ENTR is defined in equations (12) and (13),

$$
R_{i, u}(t)=\beta \cdot E\left(\log _{2} K(t)\right),
$$




$$
\begin{gathered}
F(x)=\int_{-\infty}^{x} f(t) d t=\sum_{j=1}^{L} \sum_{k=1}^{m_{j}} \frac{A_{j k}}{\sigma_{j}^{k}}\left[g\left(k-1,-\frac{1}{\sigma_{j}}, x\right)-g\left(k-1,-\frac{1}{\sigma_{j}}, 0\right)\right] . \\
K(t)=K_{1} u\left(\gamma(t)-\gamma_{1}\right)+\sum_{i=1}^{L-1}\left(K_{i+1}-K_{i}\right) u\left(\gamma(t)-\gamma_{i+1}\right), \\
R_{i, u}(t)=\beta \cdot\left\{\sum_{i=1}^{L-1} \log _{2} K_{i} \int_{C_{i}(t)}^{C_{i+1}(t)} f(x) d x+\log _{2} K_{L} \int_{C_{L}(t)}^{\infty} f(x) d x\right\} \\
=\beta \cdot\left\{\sum_{i=1}^{L-1} \log _{2} K_{i}\left[F\left(C_{i+1}(t)\right)-F\left(C_{i}(t)\right)\right]+\log _{2} K_{L}\left[1-F\left(C_{L}(t)\right)\right]\right\}
\end{gathered}
$$

where $\beta$ is a scalar that takes into account the rate loss when OSTBC is used. Note that for $2 \times 2$ Alamouti coding, $\beta=1$. In (13), $K_{i}$ is the number of the constellation points for the $i$ th modulation scheme, and $L$ is the total number of modulation schemes used in the system. Defining $C_{i}(t)=\frac{\gamma_{i}}{\rho} d_{i, u}^{2 \alpha}(t)$, it is straightforward to show that the expression for the ETNR of the AP1-UAV link can be written as in (14). A similar expression can be obtained for the UAV-AP2 link.

Since the communication between AP1 and AP2 is through the UAV relay, and also due to the two-hop half-duplex characteristics of the communication system, the overall transmission rate is

$$
R(t)=\frac{1}{2} \min \left\{R_{1, u}(t), R_{2, u}(t)\right\} .
$$

In order to improve the system performance, i.e. to increase the overall network transmission rate $R(t)$, we position the UAV according to the following optimization problem:

$$
\begin{array}{cl}
\underset{x_{u}(t), y_{u}(t), h_{u}(t)}{\arg \max } & R(t) \quad \text { s.t. } \\
& P_{e}(1, u) \leq P_{e T} \\
& P_{e}(2, u) \leq P_{e T},
\end{array}
$$

where $P_{e}(i, u) i=1,2$ is the SER between the $i$ th AP and the UAV, and $P_{e_{T}}$ is the quality of service (QoS) criterion. However due to the specific method we have used to select the thresholds for adaptive modulation, the inequality constraints in (16) can be removed.

\section{B. SER analysis}

This section is dedicated to the derivation of the SER analysis at the link level. Once the error analysis for each link has been performed, the SER of the whole system can be calculated.

1) Closed form SER expression: The SER can be expressed as in (17). In [14], an alternative definite integral form for the Gaussian Q-function is given as

$$
Q(x)=\frac{1}{\pi} \int_{0}^{\frac{\pi}{2}} \exp \left(-\frac{x^{2}}{2 \sin ^{2} \theta}\right) d \theta, \quad x \geq 0 .
$$

Using this alternative form and interchanging the order of the integrations, the SER can be rewritten as in (19). Recalling the definition in (10), it is straightforward to derive the SER expression given in (20).

2) SER upper bound: In order to relieve the computational burden when evaluating (20), an upper bound for the SER is derived by resorting to the results of [15]. In Chiani's work, an improved exponential bound for $\mathrm{Q}$ function is given as:

$$
Q(x) \leq \frac{1}{2} \sum_{i=1}^{N} a_{i} \exp \left(-\frac{b_{i} x^{2}}{2}\right),
$$

where

$$
a_{i}=\frac{2\left(\theta_{i}-\theta_{i-1}\right)}{\pi},
$$

and

$$
b_{i}=\frac{1}{\sin ^{2} \theta_{i}} .
$$

Note that this bound is much better than the popular Chernoff bound. After some manipulation, the upper bound of the SER is found to be given by (24).

\section{Numerical Simulations}

In this section, numerical simulations are conducted on both the link level (i.e. AP - UAV) and system level (i.e. AP1 UAV - AP2). In the simulations, we assume both APs and the UAV are equipped with 2 antennas, and that Alamouti coding is used. The transmit power at both AP1 and the $\mathrm{UAV}$ is $1 \mathrm{~W}$, and the noise power density at both AP2 and the UAV is $10^{-16} \mathrm{~W} / \mathrm{Hz}$. The carrier frequency of the system is assumed to be $1 \mathrm{GHz}$ and the system bandwidth is assumed to be $100 \mathrm{kHz}$. This can be seen as consistent with a narrowband system in a suburban area [6]. Seven different MPSK modulation schemes are used in the simulations, i.e. from BPSK to 128PSK. The QoS is chosen such that the average SER is less than $10^{-2}$. Note that the altitude of the $\mathrm{UAV}$ is fixed at $600 \mathrm{~m}$ in the simulations. 


$$
\begin{aligned}
P_{s}=\sum_{i=1}^{N-1} \int_{C_{i}(t)}^{C_{i+1}(t)} \bar{N}_{e}(i) \cdot Q\left(\sqrt{\frac{x \rho d_{\min }^{2}(i)}{2}}\right) f(x) d x+\int_{C_{N}(t)}^{\infty} \bar{N}_{e}(N) \cdot Q\left(\sqrt{\frac{x \rho d_{\min }^{2}(i)}{2}}\right) f(x) d x \\
P_{s}=\frac{1}{\pi}\left(\sum_{i=1}^{N-1} \int_{0}^{\frac{\pi}{2}} \int_{C_{i}(t)}^{C_{i+1}(t)} \bar{N}_{e}(i) \cdot \exp \left(-\frac{x \rho d_{\min }^{2}(i)}{4 \sin ^{2} \theta}\right) f(x) d x \cdot d \theta+\int_{0}^{\frac{\pi}{2}} \int_{C_{N}(t)}^{\infty} \bar{N}_{e}(N) \cdot \exp \left(-\frac{x \rho d_{\min }^{2}(i)}{4 \sin ^{2} \theta}\right) f(x) d x \cdot d \theta\right)(19) \\
P_{s}=\frac{1}{\pi}\left\{\sum_{i=1}^{N-1} \int_{0}^{\frac{\pi}{2}} \sum_{j=1}^{p} \sum_{k=1}^{m_{j}} \frac{\bar{N}_{e}(i) A_{j k}}{\sigma_{j}^{k}}\left[g\left(k-1,-\left(\frac{\rho d_{\min }^{2}(i)}{4 \sin ^{2} \theta}+\frac{1}{\sigma_{j}}\right), C_{i+1}(t)\right)-g\left(k-1,-\left(\frac{\rho d_{\min }^{2}(i)}{4 \sin ^{2} \theta}+\frac{1}{\sigma_{j}}\right), C_{i}(t)\right)\right] d \theta\right. \\
\left.-\int_{0}^{\frac{\pi}{2}} \sum_{j=1}^{p} \sum_{k=1}^{m_{j}} \frac{N_{e}(N) A_{j k}}{\sigma_{j}^{k}} g\left(k-1,-\left(\frac{\rho d_{\min }^{2}(N)}{4 \sin ^{2} \theta}+\frac{1}{\sigma_{j}}\right), C_{N}(t)\right) d \theta\right\} \\
P_{s} \leq \sum_{i=1}^{N-1} \frac{N_{e}(i)}{2} \sum_{j=1}^{p} \sum_{k=1}^{m_{j}} \sum_{n=1}^{Q} \frac{a_{n} A_{j k}}{\sigma_{j}^{k}}\left[g\left(k-1,-\left(\frac{b_{n} \rho d_{\min }^{2}(N)}{4}+\frac{1}{\sigma_{j}}\right), C_{i+1}(t)\right)-g\left(k-1,-\left(\frac{b_{n} \rho d_{\min }^{2}(N)}{4}+\frac{1}{\sigma_{j}}\right), C_{i}(t)\right)\right] \\
-\frac{\bar{N}_{e}(N)}{2} \sum_{j=1}^{p} \sum_{k=1}^{m_{j}} \sum_{n=1}^{Q} \frac{a_{n} A_{j k}}{\sigma_{j}^{k}} g\left(k-1,-\left(\frac{b_{n} \rho d_{\min }^{2}(N)}{4}+\frac{1}{\sigma_{j}}\right), C_{N}(t)\right)
\end{aligned}
$$

We assume rich multipath scenarios at both APs, so that the correlation matrices at the AP-side are given by

$$
\mathbf{R}_{A P i}=\left[\begin{array}{ll}
1 & 0 \\
0 & 1
\end{array}\right], \quad i=1,2 .
$$

At the UAV side, high spatial correlation is assumed:

$$
\mathbf{R}_{U A V}=\left[\begin{array}{cc}
1 & 0.8 \\
0.8 & 1
\end{array}\right]
$$

\section{A. Data rate simulation results}

In this section, we investigate overall system performance. We assume both APs are moving on circles centered at $(0,0,0)$ and $(1000,1000,0)$ respectively. The speeds for AP1 and AP2 are set at $20 \mathrm{~m} / \mathrm{s}$ and $30 \mathrm{~m} / \mathrm{s}$, with angular velocities $0.1 \mathrm{rad} / \mathrm{s}$ and $0.2 \mathrm{rad} / \mathrm{s}$ respectively. The path-loss exponent is assumed to be 1.5 and 1.7 for the links between AP1 - UAV and UAV - AP2, respectively. The optimal position of the UAV is then obtained by solving the optimization problem (16).

The trajectory of the optimal positions of the UAV is plotted in Fig. 2. It can be seen that the UAV always flies closer to AP2, due to the larger path-loss exponent for the AP2 link. The overall system and link data rates are plotted in Fig. 3, along with the theoretical system data rate. From Fig. 3, we conclude that the theoretical results match the simulated results well. Furthermore, the data rates for both links are close to each other, which indicates the proposed optimization method reaches the desired balance between them.

We also studied the influence of the path loss exponent $\alpha$ on the system performance. In this simulation, the path loss exponent is fixed at 1.5 between AP1 and UAV. From UAV to AP2, $\alpha$ is varied between 1 and 2. During the simulation,

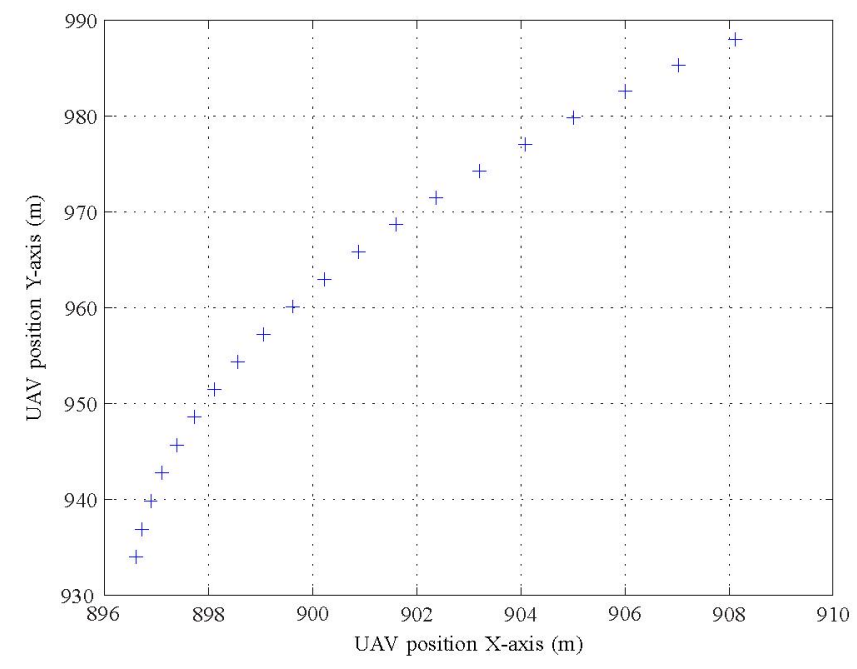

Fig. 2. Trajectory of UAV movement

the APs are fixed at $(0,0,0)$ and $(1000,1000,0)$ respectively. Fig. 4 shows the average system data rate for different $\alpha$. We find that for $\alpha=(1,1.1,1.2,1.3)$, the system data rates are the same. This is because the UAV is positioned right above AP1 for all these $\alpha$, and the system data rate is limited by the link between the AP1 and the UAV.

\section{B. SER simulation results}

In this section, we simulate a scenario in which the AP and UAV are $1.6 \mathrm{~km}$ away, and each of them is equipped with 2 antennas. The path-loss exponent is 1.5 , and we run $10^{5}$ channel realizations to simulate the SER. The transmit 

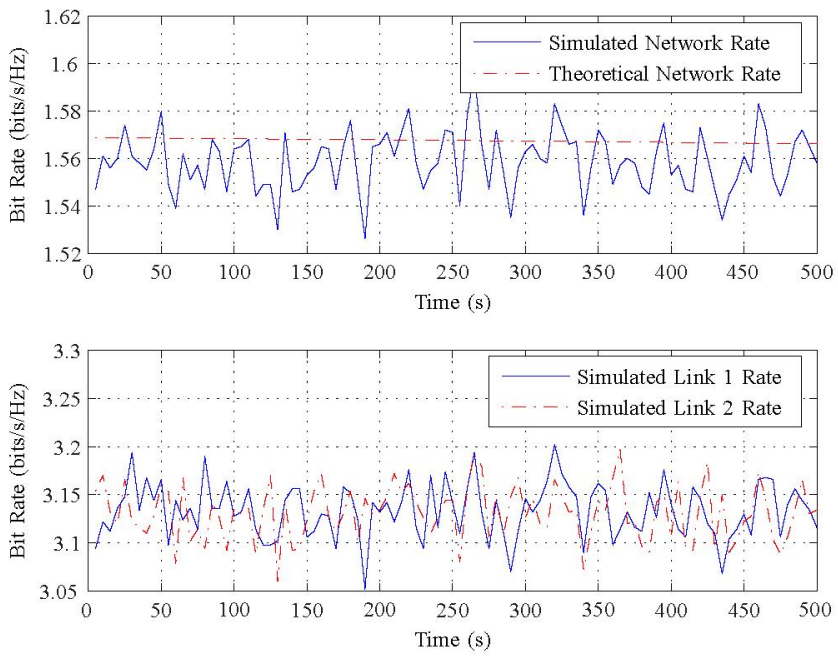

Fig. 3. System and link data rates



Fig. 4. Averaged system data rate with different $\alpha$

power for the AP is $0.1 \mathrm{~W}$, and the noise power density and system bandwidth are assumed to be the same as in the previous simulations. In Fig. 5, it is clearly shown that the derived theoretical SER match the simulation results very well. Furthermore, it shows the derived upper bound using the improved exponential bounds is quite tight in our simulation, and better than the conventional Chernoff bound.

\section{Conclusions}

In this paper, we have proposed a method to optimally deploy a UAV to improve the quality of communications between two obstructed APs. The optimal positions of the UAV have been pursued by resorting to a min-max optimization method. Closed-form expressions for the average data rate and SER have also been derived in the paper. An upper bound for the SER is also derived for the sake of reducing the required computational load. The simulation and theoretical results are shown to match very well.



Fig. 5. Link level SER

\section{REFERENCES}

[1] K. Xu, X. Hong, M. Geerla, H. Ly, and D. L. Gu, "Landmark routing in large wireless batlefield networks using UAVs," in IEEE MILCOM 2001, September 2005, vol. 1, pp. 561-573.

[2] P. Zhan, D. Casbeer, and A. Lee Swindlehurst, "A centralized control algorithm for target tracking with UAVs," in 39th IEEE Asilomar Conference, October 2005.

[3] Z. Han, A. Lee Swindlehurst, and K. J. Ray Liu, "Smart deployment/movement of unmanned air vehicle to improve connectivity in MANET," in IEEE Wireless Communications and Networking Conference, 2006, to appear.

[4] G. Kramer, M. Gastpar, and P. Gupta, "Cooperative strategies and capacity theorems for relay networks," IEEE Transactions on Information Theory, vol. 51, no. 9, pp. 3037-3063, 2005.

[5] H. Bölcskei, R. U. Nabar, O. Oyman, and A. J. Paulraj, "Capacity scaling laws in MIMO relay networks," IEEE Transactions on Wireless Communications, 2006, to appear.

[6] T. S. Rappaport, Wireless Communications, Principles and Practice, Prentice Hall PTR, 1996.

[7] D-S. Shiu, G. J. Foschini, M. J. Gans, and J. M. Kahn, "Fading correlation and its effect on the capacity of multielement antenna systems," IEEE Transactions on Communications, vol. 48, no. 3, pp. 502-513, March 2000

[8] K. Yu, M. Bengtsson, B. Ottersten, D. McNamara, P. Karlsson, and M. Beach, "Modeling of wideband MIMO radio channels based on NLOS indoor measurements," IEEE Transactions on Vehicular Technology, vol. 53, no. 3, pp. 655-665, May 2004.

[9] John G. Proakis, Digital Communications, McGraw Hill, 2001.

[10] A. Paulraj, R. Nabar, and D. Gore, Introduction to Space-Time Wireless Communications, Cambridge, 2003.

[11] V. Tarokh, H. Jafarkhani, and A. R. Calderbank, "Space-time block codes from orthogonal designs," IEEE Transactions on Information Theory, vol. 45, no. 5, pp. 1456-1467, July 1999.

[12] S. M. Alamouti, "A simple transmit diversity technique for wireless communications," IEEE Journal on Selected Areas in Communications, vol. 16 , no. 8, pp. 1451-1458, 1998.

[13] R. U. Nabar, H. Bölcskei, and A. J. Paulraj, "Outage properties of spacetime block codes in correlated Rayleigh or Ricean fading environments," in IEEE International Conference on Acoustics, Speech, and Signal Processing, 2002, vol. 3, pp. 2381-2384.

[14] M.K. Simon and M-S Alouini, "A unified approach to the performance analysis of digital communication over generalized fading channels," Proceedings of The IEEE, vol. 86, no. 9, pp. 1860-1877, 1998.

[15] M. Chiani, D. Dardari, and M. K. Simon, "New exponential bounds and approximations for the computation of error probability in fading channels," IEEE Transactions on Wireless Communications, vol. 2 , no. 4 , pp. $840-845$, July 2003. 